\title{
Diamond and $\beta$-tin structures of Si studied with quantum Monte Carlo calculations
}

\author{
D. Alfè̀ $\mathrm{e}^{1,2}$ and M. J. Gillan ${ }^{2}$ \\ ${ }^{1}$ Department of Earth Sciences, University College London \\ Gower Street, London WC1E 6BT, UK \\ ${ }^{2}$ Department of Physics and Astronomy, University College London \\ Gower Street, London WC1E 6BT, UK \\ M. D. Towler and R. J. Needs \\ Cavendish Laboratory, University of Cambridge, Cambridge CB3 0HE, UK
}

\begin{abstract}
We have used diffusion quantum Monte Carlo (DMC) calculations to study the pressure-induced phase transition from the diamond to $\beta$-tin structure in silicon. The calculations employ the pseudopotential technique and systematically improvable B-spline basis sets. We show that in order to achieve a precision of $1 \mathrm{GPa}$ in the transition pressure the non-cancelling errors in the energies of the two structures must be reduced to $30 \mathrm{meV} /$ atom. Extensive tests on system size errors, non-local pseudopotential errors, basis-set incompleteness errors, and other sources of error, performed on periodically repeated systems of up to 432 atoms, show that all these errors together can be reduced to well below $30 \mathrm{meV} /$ atom. The calculated DMC transition pressure is about 3 - $4 \mathrm{GPa}$ higher than the accepted experimental range of values, and we argue that the discrepancy may be due to the fixed-node error inherent in DMC techniques.
\end{abstract}

\section{INTRODUCTION}

The importance of the quantum Monte Carlo technique (QMC) for computing the energetics of condensed matter is becoming ever more widely appreciated. Even though its computational demands are much greater than those of standard density functional theory (DFT), its considerably greater accuracy for many systems [1] makes the additional effort well worthwhile. Indeed, QMC is often seen as one of the key ways of assessing the inadequacies of DFT [2-4]. Nevertheless, QMC itself is not exact, and it is important to probe its accuracy for different kinds of problem. A sensitive way of doing this is to examine the relative energies of different crystal structures of a material. We present here a QMC study of the energetics of the diamond and $\beta$-tin structures of silicon; we calculate their total energies as a function of volume, and hence the transition pressure between the structures, for which there are experimental data $[5,6]$. We analyse in detail the sources of the QMC errors, and use the comparison with experiment to gauge the likely size of errors that cannot be eliminated.

The QMC calculations are performed within periodic boundary conditions. Only the valence electrons are treated explicitly, the interactions between valence and core electrons being represented by pseudopotentials. We perform two type of QMC calculations: variational Monte Carlo (VMC) and diffusion Monte Carlo (DMC). DMC results are considerably more accurate, but VMC plays an indispensable role because it provides the optimised trial many-electron wavefunctions needed in DMC. This set of techniques is described in detail in a recent review [1], and implemented in the CASINO code [7] used in this work. The techniques are known to give cohesive energies for group IV elements in the diamond structure in very close agreement (within $100 \mathrm{meV} /$ atom) with experimental values. They have also indicated substantial DFT errors in, for example, the formation energy of self-interstitials in Si [3], the energetics of $\mathrm{H}_{2}$ dissociation on $\mathrm{Si}(001)$ [2], and the energies of carbon clusters [4,8].

The primary quantity calculated in this work is the total energy per atom in the perfect crystal. This energy is subject to different kinds of error. The first kind consists of errors that can in principle be reduced below any specified tolerance, for example statistical error, time step and population control bias, basis-set error in the trial wavefunction, and error due to the limited size of the periodically repeated cell. Then there are errors that cannot be systematically eliminated, but whose size can at least be estimated by purely theoretical means. The main error of this kind comes from the so called "pseudopotential localisation approximation", which cannot be avoided in present QMC techniques based on non-local pseudopotentials [10]. Finally, there are errors that cannot be eliminated and are also difficult to assess except by comparison with experiment. There is only one error of this kind, the so called QMC "fixed-node error". Our strategy in this work will be to demonstrate that all errors of the first kind have been made negligible, do our best to estimate errors of the second kind, and then appeal to experiment to assess the fixed-node error.

We have chosen to study the diamond/ $\beta$-tin transition in Si for several reasons. First, it has been investigated by several experimental groups [5], with results that are consistent enough for the present purpose. Second, there is 
already considerable QMC experience with diamond-structure $\mathrm{Si}$, from which it is known that the cohesive energy is correct to within the experimental error of $\pm 80 \mathrm{meV} /$ atom, and the equilibrium lattice parameter and bulk modulus are also accurately reproduced $[3,24,25]$. The third and most important reason for studying this transition is that it is likely to be theoretically troublesome, because of the significant change of electronic structure. In the 4 -fold coordinated diamond structure, $\mathrm{Si}$ is a semiconductor, whereas in the 6 -fold coordinated $\beta$-tin structure it is a semimetal. The difference in the electronic exchange and correlation energies between the two phases is likely to lead to non-cancelling errors. This is manifested in the serious under-prediction of the transition pressure by the local density approximation (LDA), the predicted value of $5.7-6.7 \mathrm{GPa}[11-15]$ being only about half of the experimental value of 10.3 - 12.5 GPa [5] (in fact, a value of $8.8 \mathrm{GPa}$ for the transition pressure has also been reported by one experimental group [16], but this is thought to be an underestimate). This error is considerably reduced by the generalised gradient approximation (GGA) [14]. Essentially the same DFT errors lead to an LDA under-prediction of the Si melting temperature by $23 \%[17,18]$, reduced to $12 \%$ by the GGA [18]. The reason why such transitions are a sensitive test of QMC (or any other total-energy method) is that rather small changes in relative energies give substantial changes in the transition pressure: in $\mathrm{Si}$, an energy change of $100 \mathrm{meV}$ /atom gives a change in transition pressure of $\sim 3 \mathrm{GPa}$.

The plan of the paper is as follows. In the next Section, we summarise briefly the QMC techniques and describe in more detail the sources of error and the measures we have taken to eliminate or reduce them. Sec. 3 reports our numerical results, presenting first our extensive tests on the different kinds of error and then our results for the energies, volumes and transition pressure of the diamond/ $\beta$-tin transition and the comparison with experiment. In Sec. 4 , we discuss the implications of the work and draw conclusions.

\section{METHODS}

The VMC and DMC techniques used in this work have been described in detail in reviews [1], so here we recall rather briefly the underlying ideas and outline the sources of error that we have tried to bring under control.

The VMC method gives an upper bound on the exact ground-state energy $E_{0}$. Given a normalised trial wavefunction $\Psi_{T}(\mathbf{R})$, where $\mathbf{R}=\left(\mathbf{r}_{1}, \mathbf{r}_{2} \ldots, \mathbf{r}_{N}\right)$ is a $3 N$-dimensional vector representing the positions of $N$ electrons, and denoting by $\hat{H}$ the many-electron Hamiltonian, the variational energy $E_{v} \equiv\left\langle\Psi_{T}|\hat{H}| \Psi_{T}\right\rangle \geq E_{0}$ is estimated by sampling the value of the local energy $E_{L}(\mathbf{R}) \equiv \Psi_{T}^{-1}(\mathbf{R}) \hat{H} \Psi_{T}(\mathbf{R})$ with configurations $\mathbf{R}$, distributed according to the probability density $\Psi_{T}(\mathbf{R})^{2}$. Our trial wavefunctions are of the usual Slater-Jastrow type:

$$
\Psi_{T}(\mathbf{R})=D^{\uparrow} D^{\downarrow} e^{J}
$$

where $D^{\uparrow}$ and $D^{\downarrow}$ are Slater determinants of up- and down-spin single-electron orbitals, and $e^{J}$ is the so called Jastrow factor, which is the exponential of a sum of one-body and two-body terms, with the latter being a parametrised function of electron separation, designed to satisfy the cusp conditions. The parameters in the Jastrow factor are varied to minimise the variance of the local energy $E_{L}$.

In practice, VMC results are not accurate enough, and one needs to use DMC. The basic idea is to compute the evolution of the many-body wavefunction $\Phi$ by the time-dependent Schrödinger equation in imaginary time $-\partial \Phi / \partial t=\left(\hat{H}-E_{T}\right) \Phi$, where $E_{T}$ is an energy offset. The equivalence of this to a diffusion equation allows $\Phi$ to be regarded as a probability distribution represented by a population of diffusing walkers. In practice, it is essential to use "importance sampling", which means computing the evolution of the function $f$ defined $f=\Phi \Psi_{T}$, where the trial wavefunction $\Psi_{T}$ is a good approximation to the true many-electron wavefunction, taken from the VMC calculations. The time evolution of $f$ is given by:

$$
-\frac{\partial f(\mathbf{R}, t)}{\partial t}=-\frac{1}{2} \nabla^{2} f(\mathbf{R}, t)+\nabla \cdot\left[\mathbf{v}_{D}(\mathbf{R}) f(\mathbf{R}, t)\right]+\left[E_{L}(\mathbf{R})-E_{T}\right] f(\mathbf{R}, t),
$$

where $\mathbf{v}_{D}(\mathbf{R}) \equiv \nabla \ln \left|\Psi_{T}(\mathbf{R})\right|$ is the $3 N$-dimensional drift velocity and $E_{L}(\mathbf{R})$, as before, is the local energy. In principle, the DMC scheme yields the exact ground state energy, but for fermion systems there is a fundamental problem. This is that $\Phi$ changes sign as $\mathbf{R}$ varies, so that it can only be treated as a probability in regions of $\mathbf{R}$-space where it does not change sign. These regions, and the nodal surfaces that defines their boundaries, are necessarily those of the trial wavefunction $\Psi_{T}$. The consequence is that the energy given by DMC is not the true ground state energy but is an upper bound because of the constraint that the nodal surface is that of $\Psi_{T}$. This gives rise to the so called "fixed-node" error, which is one of the concerns of this paper.

In summarising the technical questions that are important in this work, we focus on the implementation of DMC, since this determines the accuracy of the final results. The time evolution of the diffusing walkers is computed using 
the Green's function technique in the short-time approximation [1]. We shall present tests showing that the time step used in this approximation can be chosen to render errors negligible. For the representation of the single-electron orbitals contained in the Slater determinants $D^{\uparrow}$ and $D^{\downarrow}$, a number of basis sets have been used in previous work, including plane-waves and Gaussians. In the present work, we use a B-spline basis, also known as "blip functions", consisting of piecewise continuous localised cubic spline functions centred on the points of a regular grid. For a detailed account of this basis set, and an explanation of the great advantages of using this basis for QMC, see Ref. [19]. The key point here is that basis-set convergence is readily achieved simply by decreasing the spacing $a$ of the blip grid. Roughly speaking, if the single-electron orbitals would require a wavevector cut-off $k_{\max }$ for their representation in a plane-wave basis, then the blip-grid spacing will need to be $a \leq \pi / k_{\max }$, and rapid convergence is expected as $a$ is reduced below this value.

An important source of error in QMC calculations using periodic boundary conditions is the limited size of the repeating cell. In DFT calculations one normally studies a primitive unit cell and integrates quantities over the Brillouin zone, a procedure whose cost is proportional to the number of $k$-points sampled. This is equivalent to studying a much larger unit cell with a single $k$-point. In many-body calculations, it is not possible to reduce the problem to one within the primitive unit cell because the many-body Hamiltonian is not invariant under the translation of a single electron by a primitive lattice vector. In other words, one has to use a large simulation cell and solve at one $k$-point, and the cost is proportional to the cube of the number of electrons in the cell. This means that converging QMC calculations with respect to system size is much more costly than converging DFT ones. We follow the common practice [9] of correcting for this error by using separate DFT calculations: we add to the DMC energies the difference $\Delta E_{\Gamma \rightarrow \mathbf{k}}$ between the DFT-LDA energy calculated with a very large set of $\mathbf{k}$-points and the DFT-LDA energy calculated using the same sampling as in the DMC calculation.

In this work, we used pseudopotentials generated by both Hartree-Fock (HF) and LDA calculations on the Si atom. The non-locality that is essential in these pseudopotentials gives rise to unavoidable errors in DMC. The reason is that the diffusion equation with a non-local Hamiltonian becomes:

$$
-\frac{\partial f}{\partial t}=-\frac{1}{2} \nabla^{2} f+\nabla \cdot\left[\mathbf{v}_{D} f\right]+\frac{\left(\hat{H}-E_{T}\right) \Psi_{T}}{\Psi_{T}} f-\left\{\frac{\hat{V}_{n l} \Psi_{T}}{\Psi_{T}}-\frac{\hat{V}_{n l} \Phi}{\Phi}\right\} f,
$$

where $\hat{V}_{n l}$ is the non-local component of the pseudopotential. The last term in the equation can change its sign as time evolves, and therefore presents the same difficulties as the fermion sign problem. To avoid this difficulty one introduces the so called "localisation approximation", in which the last term in Eq. 3 is simply neglected. If the trial wavefunction $\Psi_{T}$ is close to the true (fixed node) ground state wavefunction $\Psi$, then this approximation introduces an error which is small and proportional to $\left(\Psi_{T}-\Psi\right)^{2}[10]$. This error, however, is non-variational, so it can decrease as well as increase the total energy.

We also tested the effect of adding a "core polarisation potential" (CPP) [20] to the pseudopotential. CPPs go beyond the standard pseudopotential approximation, by describing the polarisation of the atomic cores by the electrons and the other atomic cores. In the CPP approximation, the polarisation of a particular core is determined by the electric field at the nucleus from the instantaneous positions of the electrons and the other atomic cores. CPPs therefore account approximately for both dynamical core-valence correlation effects and static polarisation effects. Our implementation of CPPs within QMC calculations is described in Ref. [20], and we used the CPP parameters reported in Ref. [21].

Details of the CASINO code used in all the QMC calculations are given in Ref. [7]. In order to suppress statistical bias in the total energy, QMC calculations need to be run with a large population of walkers, and this makes it efficient to run on massively parallel machines, with parallelism achieved by distributing walkers across processors.

\section{RESULTS}

\section{A. Tests}

We present here the results of our tests on error sources; results on the diamond/ $\beta$-tin transition itself are reported in Sec. 3.2. To provide a framework for the discussion of errors, we set ourselves the target of reducing the sum of all controllable errors below $30 \mathrm{meV} /$ atom, this value being chosen because it corresponds to an error $\sim 1 \mathrm{GPa}$ in the transition pressure. The sources of controllable error are: sampling statistics, time step, blip-grid spacing, cell size, pseudopotentials, localisation approximation, and CPP. 
To ensure that sampling bias is negligible, our DMC calculations are run with a target population of 640 walkers for both crystal structures. With this number of walkers, the statistical error necessarily falls well below our threshold of $30 \mathrm{meV}$ /atom. The reason for this is that the DMC decay to the ground state occurs after $\sim 100$ steps, but the calculations need to extend over $\sim 1000$ steps to ensure complete stability. With the cell sizes used in this work and the number of walkers we employ, the statistical error after $\sim 1000$ steps is already less than $5 \mathrm{meV} /$ atom. As an illustration of this, Fig. 1 shows results from typical simulations of the diamond and $\beta$-tin structures, close to their equilibrium volumes. The rapid decay to the ground state is clear, and one also notes the stability of the walker population around the target value of 640 . For this number of walkers, the DMC calculations are efficient on up to 128 processors. Beyond this processor number, parallel scaling worsens, because fluctuations in the number of walkers start to cause inefficient load balancing.

In Fig. 2 we show tests on time step errors, performed with a cell containing 16 atoms in the $\beta$-tin structure at the volume $V=15 \AA^{3} /$ atom, which is close to the calculated DFT-LDA equilibrium volume. We tested time steps between 0.01 a.u. and 0.15 a.u., with the length of the runs chosen so that the statistical error was less than $10 \mathrm{meV} /$ atom. The results show that with a time step of $0.03 \mathrm{a} . \mathrm{u}$. the error is smaller than the target accuracy, and we therefore used a time step of 0.03 a.u. for our final calculations.

The blip-function basis set [19] was also tested with the 16 -atom $\beta$-tin cell and $V=15 \AA^{3} /$ atom. In Table I we report the values of the kinetic energy, the local potential energy and the non-local potential energy calculated using DFT, VMC and DMC, both with plane-waves (PW) and blips. The PW results were obtained using the PWSCF code [22] with a PW cutoff energy of 15 Ry. For the purpose of these tests, we did not use a Jastrow factor in the VMC calculations, so that the three energy terms should have exactly the same values in DFT and VMC. This is clearly the case for the VMC performed using PW, but there is a difference of up to $60 \mathrm{meV} /$ atom when the VMC calculations are performed using the blip representation with the natural grid $\left(a=\pi / k_{\max }\right)$. However, this difference is reduced to less than $5 \mathrm{meV} /$ atom if a grid of half the spacing is used $\left(a=\pi / 2 k_{\max }\right)$. This proves that, provided the blip grid is dense enough, the results are indistinguishable from those obtained using plane waves. For DMC calculations, a Jastrow factor has been included in the trial wavefunction, and the situation is more interesting. Even though the natural grid does not provide a perfect description of the single-particle orbitals, the DMC total energy is essentially the same as that calculated with PW. Of course, this is what one would expect in a perfect DMC calculation, since the total energy is independent of the trial wavefunction. However, with the fixed-node and pseudopotential localisation approximations, the total energy does in general show a weak dependence on the trial wavefunction.

Tests on the size of the simulation cell were performed on both the diamond and the $\beta$-tin structures, with cells containing up to 432 atoms. Since we were mainly interested in the diamond $\rightarrow \beta$-tin transition pressure, we calculated the energies at the two volumes $V=20 \AA^{3}$ /atom and $V=15 \AA^{3} /$ atom for the diamond and $\beta$-tin structures, respectively, which are both close to the calculated equilibrium volumes. As far as the transition pressure is concerned, the important quantity to test is the energy difference between the phases at the two volumes. The results of the tests are reported in Table II, where we also report the values of the energies extrapolated to infinite cell size $E_{\text {tot }}^{\infty}$ for the two structures. These are obtained by linear extrapolation to $1 / N$, with $N$ the number of atoms in the repeating cell, between the k-points corrected results obtained with the two cells containing 128 and 432 atoms. The cell size errors obtained with 128-atom cells are about $110 \mathrm{meV} /$ atom, and they are approximately the same in the two structures. This indicates that the residual size error can be regarded as a constant energy offset, which will not affect physical properties such as structural parameters and the diamond $\rightarrow \beta$-tin transition pressure, and we therefore chose to use cells containing 128 atoms.

We tested both a HF and a LDA pseudopotential. In both cases, the local part of the pseudopotential was chosen to be the $p$ angular momentum component. The tests were performed once again on the two structures with $V=15 \AA^{3}$ /atom and $V=20 \AA^{3}$ /atom for the $\beta$-tin and the diamond structures, respectively. We found that the energy differences between the two structures were 0.535(5) and 0.550(5) eV/atom for the HF and the LDA pseudopotentials respectively. The two numbers are very close, which indicates that the choice of the pseudopotential does not affect the results significantly. However, we believe that in a QMC calculation it is more consistent to use a HF pseudopotential rather than an LDA one, because the former does not build in any correlation. We therefore used the HF pseudopotential.

To test the pseudpotential localisation approximation, we performed additional calculations with the HF pseudopotential by changing the local part of the pseudopotential to the $s$ angular momentum component. Calculations were performed on the diamond structure with a 16-atom cell and $V=20 \AA^{3} /$ atom. Clearly, by changing the local part of the pseudopotential there is no guarantee that the quality of the pseudopotential does not change, therefore it is conceivable that the total energy may change simply because the pseudopotential has changed. So we have first performed a DFT-LDA calculation with this pseudopotential, and found a difference of less than $2 \mathrm{meV} /$ atom when the local part is changed from $p$ to $s$, which is extremely small for our purposes. We then performed a DMC simulation 
with the HF pseudopotential having the $s$ channel as the local part, and within a statistical error of $10 \mathrm{meV} /$ atom we found no energy difference from the calculation with the HF pseudopotential and the $p$ channel as the local part. This indicates that the error from the localisation approximation is probably less than $10 \mathrm{meV} /$ atom in this case.

As a final test on the pseudopotential, we considered the inclusion of a CPP. We expect the CPP energy to be more important in the $\beta$-tin structure, which has a smaller volume and therefore the electrons and ions are on average closer to one another. Tests were performed on both structures at the two volumes $V=15 \AA^{3} /$ atom and $V=20 \AA^{3} /$ atom for the $\beta$-tin and the diamond structures, respectively. We found that with the CPP the energy difference between the two calculations was $0.505(10) \mathrm{eV} /$ atom, which is slightly lower than the value of 0.535 (10) eV/atom obtained without the CPP. The inclusion of this correction has a small effect on the transition pressure which will be discussed in the next section.

\section{B. Results}

We now turn to the energetics of the diamond and $\beta$-tin structures and the transition pressure between them. Since the $\beta$-tin structure is body-centred tetragonal, its energy depends not only on volume, but also on the $c / a$ ratio. For each volume, we should therefore minimise the energy with respect to the $c / a$ ratio. However, using DFT-LDA calculations we found that the minimum of the energy depends rather weakly on $c / a$, and that choosing $c / a=0.54$ for all volumes of interest only affects the energy by a few meV/atom. To check that this $c / a$ ratio is also appropriate within DMC, we have performed DMC calculations at five different $c / a$ ratios for $V=15 \AA^{3} /$ atom. The results of the test are displayed in Fig. 3, where we report the DMC raw data and the $k$-points corrected results. By interpolating the DMC data, we find that the DMC minimum is at $c / a=0.554$, which is very close to the experimental value $c / a=0.552$. For comparison, we also report calculations for the same structures performed with DFT-LDA. It is clear that the dependence of the energy on the $c / a$ ratio is very similar in the two techniques, and therefore we chose to use $c / a=0.54$ for all calculations.

In Fig. 4 we report the calculated energies $E(V)$ for the two structures corrected for k-points errors. CPP corrections are not included in these results. These energy points were then used to fit the parameters of the Birch-Murnaghan equation of state:

$E=E_{0}+\frac{3}{2} V_{0} B_{0}\left[\frac{3}{4}(1+2 \xi)\left(\frac{V_{0}}{V}\right)^{4 / 3}-\frac{\xi}{2}\left(\frac{V_{0}}{V}\right)^{2}-\frac{3}{2}(1+\xi)\left(\frac{V_{0}}{V}\right)^{2 / 3}+\frac{1}{2}\left(\xi+\frac{3}{2}\right)\right]$

where $\xi=\left(3-3 B_{0}^{\prime} / 4\right), V_{0}$ is the equilibrium volume, $B_{0}$ the zero-pressure bulk modulus, $B_{0}^{\prime}$ its derivative with respect to pressure at zero pressure, and $E_{0}$ the energy minimum. The fitted curves are also reported on the same Figure. The values of the fitted parameters are reported in Table III together with DFT-LDA and DFT-GGA results and experimental data. We also report in the Table previous DMC results obtained by Li et al. [24] for the diamond structure. The agreement with the experimental data is extremely good, and is also somewhat better than obtained previously by $\mathrm{Li}$ et al [24]. In particular, the equilibrium volume is overestimated by only $0.5 \%$. In comparison, DFT-LDA underestimates the equilibrium volume by $2 \%$, and the two DFT-GGA BP and PW91 [14] overestimate it by $1 \%$ and $2 \%$, respectively.

Using our results, we obtain a DMC transition pressure of $\sim 19$ GPa. Before comparing our calculated transition pressure with the experiments, we note that our calculations do not include zero-point motion, which has been shown to be different in the two phases. Moreover, experimental transition pressures are only reported at room temperature, therefore there is a significant contribution to the free energy coming from the difference in vibrational free energies between the two structures. As shown by Gaál-Nagy et al. [11], the zero point motion stabilises the $\beta$-tin structure with respect to the diamond structure, and lowers the transition pressure by about $0.3 \mathrm{GPa}$. At room temperature the stabilisation of the $\beta$-tin structure lowers the transition pressure by an additional $1 \mathrm{GPa}$, so that the two effects lower the transition pressure by $\sim 1.3 \mathrm{GPa}$. If we add this correction to our calculated transition pressure we obtain 17.7 GPa. Moreover, these calculations did not include CPP corrections, which reduces the free energy of the $\beta$ tin structure relative to the diamond structure. If we assume that these corrections are approximately the same at different volumes in the two structure, then we finally obtain a corrected transition pressure of $16.5 \mathrm{GPa}$. The experimental transition pressure is in the range 10.3 - 12.5 GPa [5], which is significantly lower than predicted by our calculations. 


\section{DISCUSSION}

We recall that the main purpose of this work is to assess the accuracy of QMC for Si by examining its prediction for the transition pressure between the diamond and $\beta$-tin structures. However, we discuss first the controllable sources of error that we have attempted to reduce below our threshold of $30 \mathrm{meV} /$ atom.

We have shown that errors due to statistical sampling and finite time step are readily reduced to negligible size. Convergence with respect to basis set completeness is also easy to achieve, and we have noted the important advantages of the B-spline (blip) basis, which combines ease of convergence with excellent scaling with respect to system size, as reported in detail elsewhere [19]. System size errors also appear to be under excellent control. By performing DMC on cells of up to 432 atoms, we have shown that the error in total energy is reduced to $\sim 110 \mathrm{meV} /$ atom, but the size error on the difference in energy between the diamond and $\beta$-tin structures is reduced to less than $\sim 5 \mathrm{meV} /$ atom. Errors due to the pseudopotential approximation itself, as well as to the pseudopotential localisation approximation, also appear to be no larger than $\sim 5 \mathrm{meV} /$ atom, though we have not shown this rigorously. Finally, we have studied the effect of including core polarisation, and shown that this reduces the energy difference between the two structures by $\sim 30 \mathrm{meV} /$ atom. Taken together, these tests suggest that if there were no other sources of error, the transition pressure could be calculated to within $\sim 1 \mathrm{GPa}$.

Our results for diamond-Si confirm the excellent accuracy of DMC for this structure. Our cohesive energy agrees with the experimental value within the experimental error of $\sim 80 \mathrm{meV} /$ atom, the equilibrium lattice constant is correct to $0.2 \%$ and the bulk modulus to $3 \%$. However, for the transition pressure, our DMC result of $16.5 \mathrm{GPa}$ is significantly larger than the experimental range of $10.3-12.5 \mathrm{GPa}$. This less than satisfactory agreement could in principle be due either to uncertainty in the experimental results or to remaining errors in the QMC calculations. We think it unlikely that experiments could underestimate the equilibrium transition pressure by such a large amount. There appears to be a large barrier to the transition on increase of pressure, and the transition is in fact irreversible, with complex tetrahedral phases being formed on release of pressure [5,6]. If anything, this irreversibility would make it more likely for the experimental values to be too high. On the theoretical side, we have shown that most of the sources of error are too small to account for the discrepancy. The only remaining theoretical error that could be large enough is the fixed-node error. Since the fixed node error can only increase the energy, and since the DMC transition pressure is too high, a possible scenario is that the fixed-node error raises the energy of $\beta$-tin-Si relative to diamond-Si.

In conclusion, we have shown the feasibility of using QMC to calculate the relative stability of different crystal structures, with most technical errors reduced enough to give the transition pressure to within $\sim 1 \mathrm{GPa}$. Nevertheless, the computed transition pressure for the diamond $\rightarrow \beta$-tin transition in Si differs from the experimental value by $\sim 4-6 \mathrm{GPa}$. The evidence presented indicates that the discrepancy may be due to QMC fixed-node error.

\section{ACKNOWLEDGMENTS}

The computations were performed on the CSAR and the HPCx services, using allocations of time from NERC through the Mineral Physics Consortium and from EPSRC through the UKCP Consortium. Calculations have also been performed on the Altix machine at University College London provided by the SRIF programme. DA acknowledges support from the Royal Society and the Leverhulme Trust. We gratefully acknowledge helpful discussions with Cyrus Umrigar.

[1] W. M. C. Foulkes, L. Mitas, R. J. Needs, and G. Rajagopal, Rev. Mod. Phys. 73, 33 (2001).

[2] C. Filippi, S. B. Healy, P. Kratzer, E. Pehlke, and M. Scheffler, Phys. Rev. Lett. 89, 166102 (2002).

[3] W.-K. Leung, R. J. Needs, G. Rajagopal, S. Itoh, and S. Ihara, Phys. Rev. Lett. 83, 2351 (1999).

[4] J. C. Grossman, L. Mitas, and K. Raghavachari, Phys. Rev. Lett. 75, 3870 (1995); erratum: ibid. 76, 1006 (1995).

[5] J. C. Jamieson, Science 139, 762 (1963); ibid. 139, 845 (1963); J. Z. Hu, L. D. Merkle, C. S. Menoni, and I. L. Spain, Phys. Rev. B 34, 2679 (1986); M. I. McMahon and R. J. Nelmes, Phys. Rev. B 47, 8337 (1993); M. I. McMahon, R. J. Nelmes, N. G. Wright, and D. R. Allan, Phys. Rev. B 50, 739 (1994).

[6] A. Mujica, A. Rubio, A. Muñoz, and R. J. Needs, Rev. Mod. Phys. 75, 863 (2003).

[7] R. J. Needs, M. D. Towler, N. D. Drummond, and P. R. C. Kent, 'CAsino Version 1.7 User Manual', University of Cambridge, Cambridge (2004). 
[8] P. R. C. Kent, M. D. Towler, R. J. Needs, and G. Rajagopal, Phys. Rev. B 62, 15394 (2000).

[9] A. J. Williamson. R. Q. Hood, R. J. Needs, and G. Rajagopal, Phys. Rev. B, 57, 12140 (1998).

[10] M. M. Hurley and P. A. Christiansen, J. Chem. Phys. 86, 1069 (1987); B. L. Hammond, P. J. Reynolds, and W. A. Lester, Jr., J. Chem. Phys. 87, 1130 (1987); L. Mitas, E. L. Shirley, and D. M. Ceperley, J. Chem. Phys. 95, 3467 (1991).

[11] K. Gaál-Nagy, A. Bauer, M. Schmitt, K. Karch, P. Pavone, and D. Strauch, Phys. Stat. Sol. B 211, 275 (1999); K. Gaál-Nagy, M. Schmitt, P. Pavone, and D. Strauch, Comp. Mat. Sci. 22, 49 (2001).

[12] L. L. Boyer, E. Kaxiras, J. L. Feldman, J. Q. Broughton, and M. J. Mehl, Phys. Rev. Lett. 67, 715 (1991).

[13] R. J. Needs and A Mujica, Phys. Rev. B 51, 9652 (1995).

[14] N. Moll, M. Bockstedte, M. Fuchs, E. Pehlke, and M. Scheffler, Phys. Rev. B 52, 2550 (1995).

[15] The range 5.7 - 6.7 GPa for the LDA transition pressure of Si from the diamond to the $\beta$-tin structure also includes the effect of the phonon free energy at room temperature [11], which lowers the transition pressure by about 1.3 GPa with respect to the transition pressure calculated without including this effect.

[16] H. Olijnyk, S. K. Sikka, and W. B. Holzapfel, Phys. Lett. 103a, 137 (1984).

[17] O. Sugino and R. Car, Phys. Rev. Lett. 74, 1823 (1995).

[18] D. Alfè and M. J. Gillan, Phys. Rev. B 68, 205212 (2003).

[19] D. Alfè and M. J. Gillan, unpublished.

[20] R. Maezono, M. D. Towler, Y. Lee, R. J. Needs, Phys. Rev. B 68, 165103 (2003).

[21] E. L. Shirley and R. M. Martin, Phys. Rev. B 47, 15413 (1993).

[22] S. Baroni, A. Dal Corso, S. de Gironcoli, and P. Giannozzi, http://www.pwscf.org.

[23] Y. Lee and R. J. Needs, Phys. Rev. B 67, 035121 (2003).

[24] X.-P. Li, D. M. Ceperley, and R. M. Martin, Phys. Rev. B 44, 10929 (1991).

[25] P. R. C. Kent, R. Q. Hood, A. J. Williamson, R. J. Needs, W. M. C. Foulkes, and G. Rajagopal, Phys. Rev. B 59, 1917 (1999).

[26] M. T. Yin and M. L. Cohen, Phys. Rev. B 26, 5669 (1982).

[27] JANAF Thermodynamic Tables, J. Phys. Chem. Ref. Data, Vol. 14, Suppl. 1, edited by M. W. Chase, Jr., C. A. Davis, J. R. Downey, Jr., D. J. Frurip, R. A. Mcdonald, and A. N. Syverud, (American Chemical Society, Washington, D.C.), (1985).

[28] R. G. Hennig and C. J. Umrigar, private communication.

\begin{tabular}{|c|c|c|c|c|}
\hline & & PW & Blips $\left(a=\pi / k_{\max }\right)$ & $\overline{\operatorname{Blips}\left(a=\pi / 2 k_{\max }\right)}$ \\
\hline \multicolumn{5}{|r|}{ 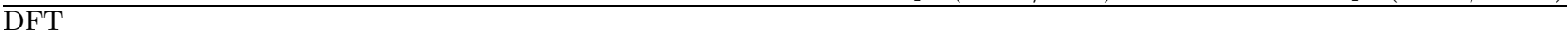 } \\
\hline & $E_{\text {kin }}$ & 43.863 & & \\
\hline & $E_{\mathrm{loc}}$ & 15.057 & & \\
\hline & $E_{\mathrm{nl}}$ & 1.543 & & \\
\hline \multicolumn{5}{|l|}{ VMC } \\
\hline & $E_{\text {kin }}$ & $43.864(3)$ & $43.924(3)$ & $43.862(3)$ \\
\hline & $E_{\mathrm{loc}}$ & $15.057(3)$ & $15.063(3)$ & $15.058(3)$ \\
\hline & $E_{\mathrm{nl}}$ & $1.533(3)$ & $1.525(3)$ & $1.535(3)$ \\
\hline & $E_{\text {tot }}$ & $-101.335(3)$ & $-101.277(3)$ & $-101.341(3)$ \\
\hline \multicolumn{5}{|l|}{ DMC } \\
\hline & $E_{\text {tot }}$ & $-105.714(4)$ & $-105.713(5)$ & $-105.716(5)$ \\
\hline
\end{tabular}

TABLE I. Total energy $E_{\text {tot }}$ and the kinetic energy, local pseudopotential energy and non-local pseudopotential energy components $E_{\text {kin }}, E_{\text {loc }}$ and $E_{\mathrm{nl}}$ calculated using plane wave (PW) basis sets and blip function basis sets with two grid spacings $a$ (energy units: eV/atom). Results are from DFT, VMC and DMC calculations on Si in the $\beta$-tin structure, with a repeating cell of 16 atoms. A Jastrow factor was included only in the DMC calculations. Blip-grid spacing $a$ is specified in terms of the PW cut-off wavevector $k_{\max }$, corresponding to a cut-off energy of $15 \mathrm{Ry}$. 


\begin{tabular}{lcccc|cccc|c}
\hline \hline \multicolumn{9}{c}{128} & \multicolumn{4}{c}{432} \\
& $E_{\Gamma}$ & $\Delta E_{\Gamma \rightarrow \mathbf{k}}$ & $E_{\text {tot }}$ & $\delta E$ & $E_{\Gamma}$ & $\Delta E_{\Gamma \rightarrow \mathbf{k}}$ & $E_{\text {tot }}$ & $\delta E$ & $E_{\text {tot }}^{\infty}$ \\
\hline diamond & $-106.926(5)$ & -0.102 & $-107.028(5)$ & $0.108(5)$ & $-106.937(6)$ & -0.014 & $-106.951(6)$ & $0.031(6)$ & $-106.920(5)$ \\
$\beta$-tin & $-106.457(5)$ & -0.045 & $-106.502(5)$ & $0.117(5)$ & $-106.416(7)$ & -0.004 & $-106.420(7)$ & $0.035(7)$ & $-106.385(5)$ \\
\hline \hline
\end{tabular}

TABLE II. DMC total energies (eV/atom) for the diamond and the $\beta$-tin structures of Si, obtained from calculations on 128-atom and 432-atom cells. $E_{\Gamma}$ is the raw DMC energy, $\Delta E_{\Gamma \rightarrow \mathbf{k}}$ is the k-points correction, $E_{\text {tot }}$ is the sum of $E_{\Gamma}$ and $\Delta E_{\Gamma \rightarrow \mathbf{k}}$, and $E_{\mathrm{tot}}^{\infty}$ is the value of $E_{\mathrm{tot}}$ extrapolated to infinite cell size. For each cell size, $\delta E$ is the difference $E_{\mathrm{tot}}-E_{\mathrm{tot}}^{\infty}$.

\begin{tabular}{|c|c|c|c|c|c|c|}
\hline & Expt. & LDA & $\mathrm{BP}$ & PW91 & DMC & $\overline{\text { DMC (This work) }}$ \\
\hline \multicolumn{7}{|l|}{$\overline{\text { Diamond }}$} \\
\hline$V_{0}\left(\AA^{3}\right)$ & $20.01^{a}$ & $19.57^{b}$ & $20.46^{b}$ & $20.23^{b}$ & $20.23(20)^{c}$ & $20.11(3)$ \\
\hline$B_{0}(\mathrm{GPa})$ & $99^{a}$ & $97^{b}$ & $90^{b}$ & $92^{b}$ & $103(7)^{c}$ & $103(10)$ \\
\hline$E_{c o h}(\mathrm{eV})$ & $4.62(8)^{d}$ & $5.338^{e}$ & & $4.653^{e}$ & $4.51(3)^{c}, 4.63(2)^{f}$ & $4.62(1)$ \\
\hline \multicolumn{7}{|l|}{$\beta$-tin } \\
\hline$V_{0}\left(\AA^{3}\right)$ & & $14.63^{b}$ & $15.84^{b}$ & $15.67^{b}$ & & $15.26(3)$ \\
\hline$B_{0}(\mathrm{GPa})$ & & $115^{b}$ & $99^{b}$ & $104^{b}$ & & $114(5)$ \\
\hline$E_{c o h}(\mathrm{eV})$ & & $5.115^{e}$ & & $4.313^{e}$ & & $4.10(1)$ \\
\hline$c / a$ & $0.552^{a}$ & $0.548^{b}$ & & $0.548^{b}$ & & 0.554 \\
\hline$\overline{\Delta E_{0}(\mathrm{eV})}$ & & $0.226^{b}$ & $0.404^{b}$ & $0.341^{b}$ & & $0.505(10)$ \\
\hline$p_{t}(\mathrm{GPa})$ & $10.3-12.5^{g}$ & $6.7^{b}$ & $13.3^{b}$ & $10.9^{b}$ & & $16.5(5)$ \\
\hline
\end{tabular}

${ }^{a}$ Cited in Ref. [26]

${ }^{b}$ Ref. [14]

${ }^{c}$ Ref. [24]

${ }^{d}$ Ref. [27]

${ }^{e}$ Ref. [28]

${ }^{f}$ Ref. [3]

${ }^{g}$ Ref. [5]

TABLE III. Structural properties and the diamond $\rightarrow \beta$-tin transition pressure $p_{t}$, calculated within DFT using different exchange-correlation functionals and within DMC. $V_{0}$ is the equilibrium volume, $B_{0}$ the zero pressure bulk modulus, $E_{c o h}$ is cohesive energy, $\Delta E_{0}$ is the calculated difference of minimum energy between the two structures, and $c / a$ is the ratio of tetragonal lattice parameters of the $\beta$-tin structure. Theoretical cohesive energies have all been corrected for zero point motion ( $0.06 \mathrm{eV}$ and $0.04 \mathrm{eV}$ in the diamond and $\beta$-tin structures, respectively). The structural parameters calculated within DMC for the diamond structure were obtained from a Birch-Murnaghan equation of state fit to energies calculated at volumes between 17 and $24 \AA^{3}$ /atom. For the $\beta$-tin structure volumes between 11 and $19 \AA^{3} /$ atom were used. The experimental data are at $0 \mathrm{~K}$ and $77 \mathrm{~K}$, for the zero pressure equilibrium volume and bulk-modulus, respectively. The transition pressures have been corrected for finite temperature effects evaluated at $300 \mathrm{~K}$ [11]. The DMC calculations for the transition pressure also include a CPP. 

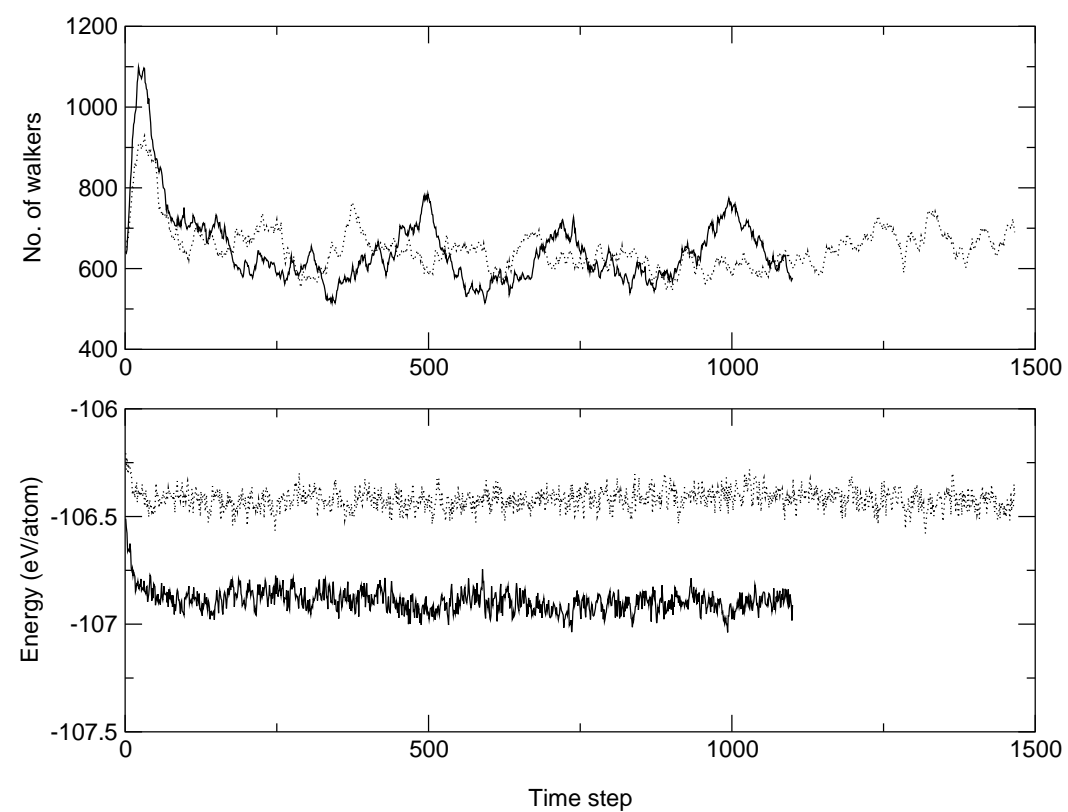

FIG. 1. Lower panel: DMC local energies as function of time (time-step=0.03 a.u.) for the $\beta$-tin structure with $V=15 \AA^{3} /$ atom (dotted line) and the diamond structure with $V=20 \AA^{3} /$ atom (continuous line). Upper panel: the population of walkers for the $\beta$-tin structure (dotted line) and the diamond structure (continuous line).

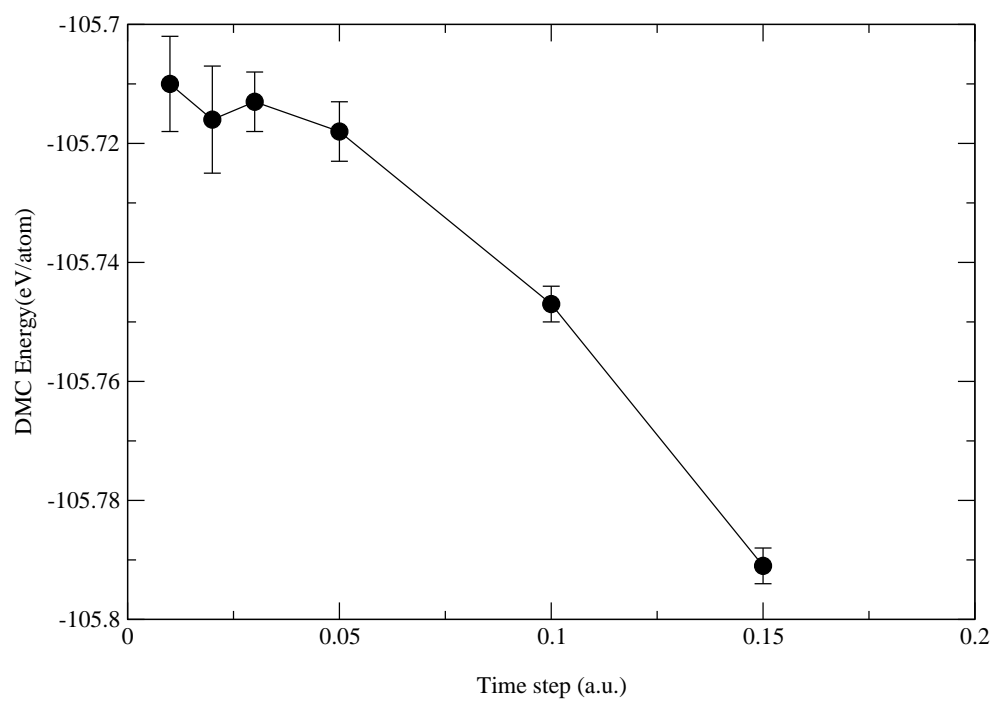

FIG. 2. The DMC total energy per atom as a function of time step, with error bars showing the statistical errors. The calculations were performed using a cell containing 16 atoms in the $\beta$-tin structure at the volume $V=15 \AA^{3} /$ atom. 


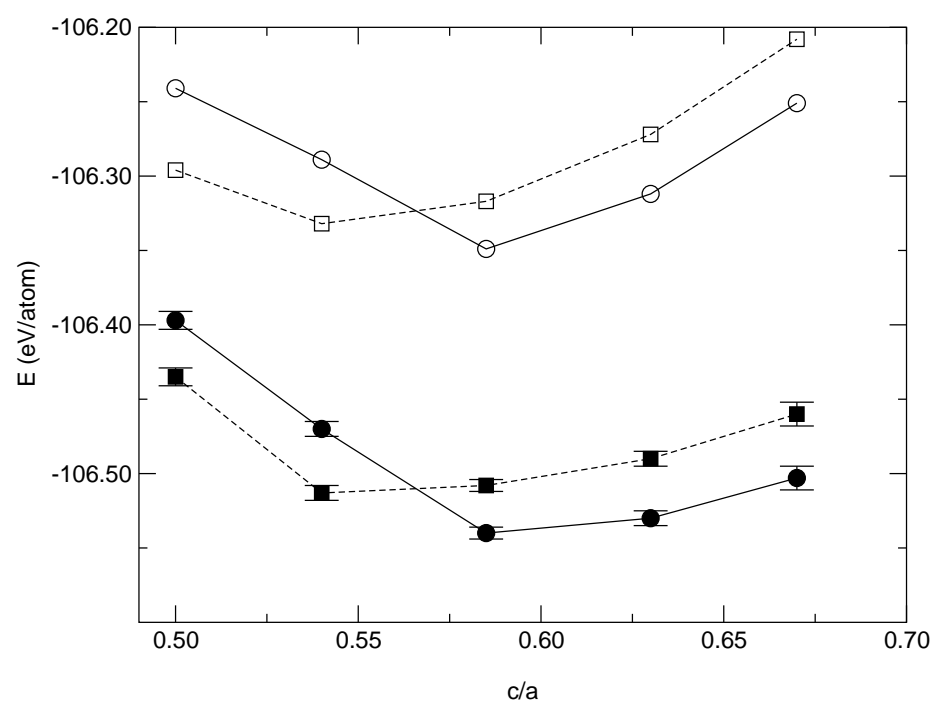

FIG. 3. DMC energies for the $\beta$-tin structure at the volume $V=15 \AA^{3} /$ atom as a function of the $c / a$ ratio, performed with cells containing 128 atoms (filled circles). DFT results performed with the equivalent mesh of k-points are also shown (open circles). DMC k-points corrected results are shown as filled squares, and DFT results fully converged with respect to k-point sampling (open squares). The lines are guides to the eye.

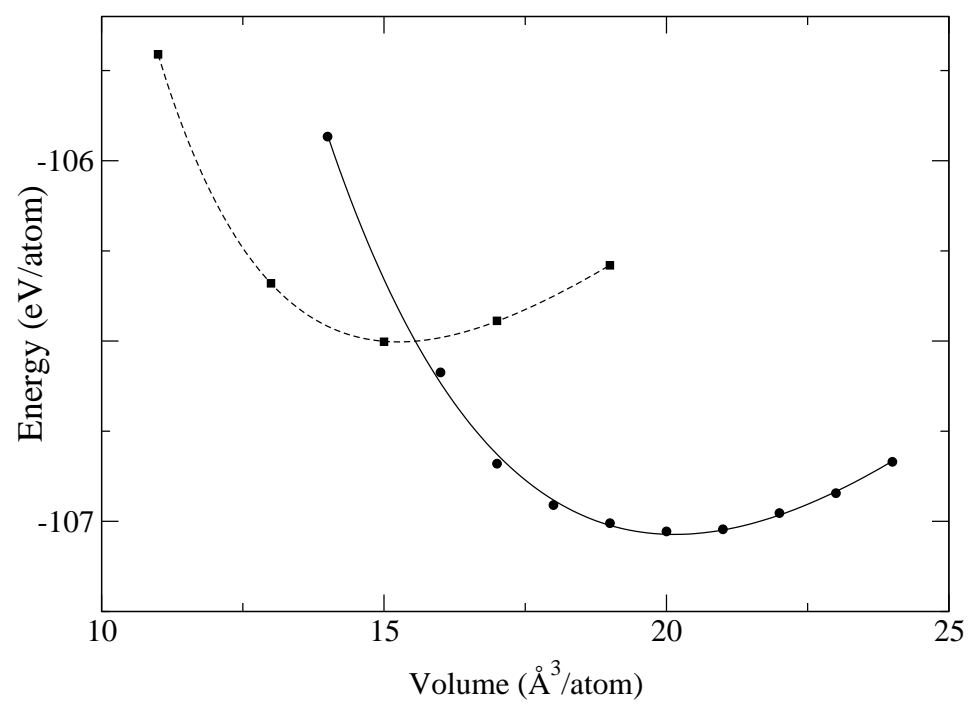

FIG. 4. DMC total energies for the $\beta$-tin (squares) and the diamond (circles) structures. The size of the points corresponds to about two standard deviations. The dashed and continuous lines are Birch-Murnaghan EOS curves fitted to the data. 